\title{
Trabajadoras del hogar: grupo vulnerable al maltrato y desigualdad laboral
}

\section{Domestic workers: vulnerable to abuse and employment inequality group}

\section{Yolanda Velázquez Narváez ${ }^{1}$ \\ Fabiola Peña Cárdenas ${ }^{2}$ \\ Lucía Ruíz Ramos ${ }^{3}$}

${ }^{1}$ Universidad Autónoma de Tamaulipas, México.

Correo electrónico: yovelazquez@docentes.uat.edu.mx

${ }^{2}$ Universidad Autónoma de Tamaulipas, México.

Correo electrónico: fcardenas@docentes.uat.edu.mx

${ }^{3}$ Universidad Autónoma de Tamaulipas, México.

Correo electrónico: luruiz@docentes.uat.edu.mx

\begin{abstract}
Resumen
El presente trabajo surge como producto de la red de investigación

"Evaluación e intervención en factores de riesgos psicosociales" y tiene dos objetivos; el primero consiste en establecer las condiciones de empleo de las trabajadoras del servicio doméstico en México y la percepción que tienen respecto al trato de sus patrones hacia ellas; y el segundo en estimar la probabilidad de que la empleada doméstica perciba un buen trato por parte de sus patrones, de acuerdo con cada una de las variables que componen los modelos de estudio.

Para ello, se tomó información de una fuente secundaria a partir de la Encuesta Nacional sobre Discriminación en México (ENADIS, 2010),
\end{abstract}


YOLANDA VELÁZqUEZ NARVÁEZ / FABIOLA PEÑa CÁRDENAS / LUCÍA RUÍZ RAMOS

aplicada a trabajadoras del servicio doméstico. La muestra estuvo conformada por 404 mujeres trabajadoras domésticas en la República Mexicana. Se encontró que un elevado porcentaje de empleadas domésticas no cuentan con prestaciones laborales, y que el $23.7 \%$ de la muestra consideran que no son tratadas con respeto, al menos en ocasiones. La única variable respecto a las características contractuales de trabajo asociada al trato con respeto fue el aguinaldo, mientras que, para las características del trato, las variables asociadas fueron uso del teléfono y permiso de salir.

Se concluye que la desigualdad de género y discriminación hacia la mujer trabajadora en México sigue siendo un problema latente, vulnerando especialmente a las trabajadoras del hogar, donde se presenta nulidad de sus derechos legales como trabajadoras y situaciones de discriminación y maltrato. No obstante, muchas veces ellas no son conscientes de esta situación. Por otro lado, la percepción que tienen en cuanto al trato con respeto que reciben por parte de sus empleadores no se asocia directamente a las características contractuales de trabajo, sino al grado de libertad percibido para atender situaciones personales durante su jornada laboral.

Palabras clave: empleadas del hogar, desigualdad laboral, género, violencia laboral, maltrato

\section{Abstract}

This work was originated by the "evaluation and intervention on psychosocial risks factors project" and has two objectives; the first is to 
establish the conditions of employment of women workers in domestic service in Mexico and her perceptions with respect to treatment from her employers, and the second is estimating the probability that the domestic employee perceives a good treatment by their employers, according to each of the variables that compose the study models.

The information was taken from a secondary source from the National Survey of Discrimination in Mexico (ENADIS, 2010), applied to domestic service workers. The sample was 404 women domestic workers in the Mexican Republic. It was found that a high percentage of domestic workers don't have employment benefits, and the $23.7 \%$ of the sample consider that they are not treated with respect, at least occasionally. The only variable for the contractual characteristics of work associated with dealing with respect was the bonus, while for the characteristics of the treatment; the associated variables were using the phone and permission to leave.

It is concluded that the gender inequality and discrimination against women workers in Mexico is still a latent problem, especially present in the domestic workers, where nullity of legal protection generate discrimination and ill treatment. However, often they are not aware of this situation. On the other hand, the perception regarding dealing with respect they receive from their employers is not associated directly contractual characteristics of work, but with the perceived degree of freedom to respond to personal situations during their workday.

Keywords: domestic employees, occupational inequality, gender, labor violence, ill treatment 


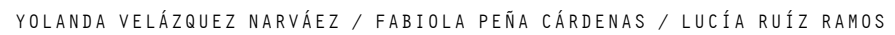

RECEPCIÓN: 02 DE AGOSTO DE 2018/ACEPTACIÓN: 16 DE OCTUBRE DE 2018

\section{Problemática de desigualdady maltrato que viven las traba-} jadoras domésticas

A través de la historia se ha podido constatar que las mujeres latinoamericanas suelen ser continuamente víctimas de violencia laboral. "Las conductas abusivas de algunos empleadores y empleadoras se manifiestan en malos tratos y en la negación de sus derechos" (CONAPRED, 2011, p. 50).

Por lo tanto, la violencia emerge desde dos vertientes que, aunque son independientes una de la otra, se relacionan estrechamente entre sí. Una se encuentra asociada a conductas agresivas contra la mujer trabajadora expresadas por maltrato físico o verbal, hostigamiento y acoso sexual, generada principalmente por sus patrones o supervisores inmediatos; mientras que la otra es más sistematizada, ya que surge a partir de la falta de protección legal (o vigilancia de su cumplimiento) hacia sus derechos como trabajadoras, originando actos de discriminación y desigualdad laboral, manifestadas por jornadas laborales arbitrarias, falta de prestaciones sociales, salarios poco competitivos y amplia rotación (Anguiano y Ortiz, 2013, p. 97). En ambos casos, predomina una violencia de género, en la cual las mujeres integran el grupo más vulnerable (Maqueda, 2006, p. 8).

Esta expresión de discriminación y desigualdad se refleja en las condiciones laborales que caracterizan la inserción cada vez más 
frecuente de la mujer en el campo productivo, donde las condiciones de trabajo son nada competitivas, y predomina una falta de acceso a trabajos más calificados, originando no sólo condiciones laborales subestándares, sino además, propiciando que dichas mujeres sean un blanco fácil de la victimización de la violencia en el ámbito laboral, al carecer de condiciones de equidad respecto al género masculino (Alonso et al., 2006, p. 156).

Uno de los grupos laborales altamente vulnerables es sin duda el de las trabajadoras del hogar, en el cual el nivel de ingreso es muy inferior respecto a otras ocupaciones (Horbath y Gracia, 2014, p. 478). La Organización Internacional del Trabajo (en delante OIT) (2010a, p. 11), define el trabajo doméstico desde 1951 como: "un trabajador remunerado que trabaja en un domicilio privado, sea cual sea el método y el período de remuneración, que pueda estar empleado por uno o varios empleadores que no obtienen ningún beneficio pecuniario por esa labor”. Daeren (2000, p. 8), asevera que esta ocupación históricamente se ha caracterizado por condiciones de trabajo precarias, ya que suele presentarse un elevado grado de dependencia entre la empleada y el empleador, predominando un estado constante de subordinación. También afirma que en este sector productivo existe una exclusión de la legislación laboral y que este problema aumenta con el hecho de que muchas mujeres que ocupan estos puestos son migrantes.

En este sentido, la OIT (2018, p. 1-2), considera que el personal doméstico remunerado pertenece al grupo de trabajadores asalariados informales que se ubican afuera del sector informal, sin em- 
bargo, le reconoce a esta ocupación los derechos de cualquier otra actividad económica (OIT, 2010a, p. 1). De hecho, en el año 2011, la OIT impulsó el Convenio 189 sobre la regulación y protección de los trabajadores domésticos (Reynoso, 2011, p. 593). Sin embargo, éste no ha sido ratificado por México, por lo que puede entenderse que "el Estado Mexicano no reconoce los derechos de las mujeres que trabajan en el hogar" (Santillán, 2016, p. 69). No obstante existen esfuerzos localizados por empoderar a las mujeres trabajadoras de este sector productivo, de tal forma que, desde el año 2000 se creó el Centro de Apoyo y Capacitación para Empleadas del Hogar (CACEH), el cual funciona en la Ciudad de México, y más recientemente (2015), el Sindicato Nacional de Trabajadores y Trabajadoras del Hogar (SINACTRAHO) (Rojas y Contreras, 2018 , p. 3). A pesar de ello, la realidad que viven en el día a día las mujeres que integran este grupo laboral, refleja una serie de situaciones que merman su calidad de vida como trabajadoras, haciéndolas objeto de diferentes formas de discriminación.

Ante esto, las autoras del presente trabajo se proponen lograr dos objetivos principales durante el desarrollo del mismo. El primero, consiste en establecer las condiciones de empleo de las trabajadoras del servicio doméstico en México y la percepción que tienen respecto al trato de sus patrones hacia ellas, para lo cual se utilizaron los datos generados por la Encuesta Nacional sobre Discriminación en México (ENADIS, 2010). El segundo, consiste en estimar la probabilidad de que la empleada doméstica perciba un buen trato por parte de sus patrones, de acuerdo con cada una de las varia- 
bles que componen los modelos de estudio, las cuales consideran aspectos tanto del grado de libertad y confianza percibidos por la empleada, como características del salario y prestaciones recibidas como remuneración a su trabajo.

\section{El estado de las mujeres trabajadoras del hogar en México}

De acuerdo con cifras oficiales del Instituto Nacional de Geografía y Estadística - INEGI (2017, pp. 2-13), en México el 51.4\% de la población son mujeres. Para el tercer semestre del año 2016, las mujeres de 15 años y mayores era de 19.9 millones, las cuales en su mayoría se encontraban laboralmente ocupadas. Se considera que dos de cada tres de ellas son subordinadas y remuneradas. Sobre este fenómeno, Barrios y Barrios (2016, p. 43), afirman que, durante el primer trimestre del 2016, la fuerza laboral de las mujeres mexicanas se concentró principalmente en el sector de servicios con una participación del $53.8 \%$ de las mujeres empleadas.

Sin embargo, el $37.7 \%$ no cuenta con acceso a servicios de salud como parte de sus prestaciones laborales, el 41.9\% no cuenta con un contrato escrito, el $33.8 \%$ no goza de prestaciones laborales, sólo una de cada dos trabajadoras subordinadas tiene derecho a vacaciones pagadas, poco más de la mitad (62.6\%) recibe aguinaldo y únicamente el $16.9 \%$ recibe reparto de utilidades (INEGI, 2017, pp. 2-13). Esto implica una situación permanente de desventaja social y económica ante la falta de protección laboral, además de no coincidir con los principios del concepto de trabajo decente 
(Ghai, 2003, p. 125), el cual es concebido como un "trabajo productivo y adecuadamente remunerado, ejercido en condiciones de libertad, equidad, seguridad y dignidad humana" (Barrón, 2013, p. 109), ya que cuando se compara la inserción de las mujeres al sector productivo en relación a la inserción de los hombres, se evidencian diversas formas de discriminación que van desde la segregación ocupacional, hasta la diferenciación en cuanto al salario percibido y el acceso a programas sociales y de salud (Horbat y García, 2014, p. 486). De tal forma que este grupo laboral es altamente vulnerable tanto en la dimensión social como económica. Esta vulnerabilidad se origina en la subvaloración del trabajo femenino y del rol social que se le ha asignado a la mujer a través de la historia (Rodgers, 2009, p. 71).

Esta problemática se acentúa en los grupos de mujeres indígenas jóvenes, que al tener que migrar hacia áreas urbanas, ingresan al campo productivo casi automáticamente como empleadas domésticas (Horbat, 2008, p. 34). De acuerdo con Mendoza y Armendáriz (2017, p. 2), el 11\% del total de mujeres empleadas en los hogares mexicanos son de origen indígena. En este sentido, Hidalgo (2016, p. 6) establece que esta inclusión al campo productivo por medio de la contratación de servicios para el hogar, representa en sí misma una condición de discriminación hacia estas mujeres, al ser una elección que es resultado de la falta de oportunidades laborales.

Para Lexartza, Chaves y Carcedo (2016, p. 7), el trabajo doméstico es considerado como una de las ocupaciones más afectadas por 
la informalidad en los países de América Latina y el Caribe, ocupando alrededor de 18 millones de personas en la región, de las cuales el $93 \%$ son mujeres. No obstante, a pesar de ser una de las principales fuentes de empleo femeninas, sigue siendo uno de los espacios laborales con mayores desventajas respecto a otros empleos, es decir, a pesar de los esfuerzos realizados por los gobiernos para fortalecer la igualdad de género y el derecho al trabajo digno, el servicio doméstico continúa siendo subvalorado, en su calidad de trabajo no cualificado, puesto que se parte de la idea de que la empleada, por el hecho de ser mujer, tiene la capacidad innata de desempeñar las tareas del hogar (OIT, 2010b, p. 5).

Durin (2013, p. 104) señala que existen algunas características comunes entre las trabajadoras domésticas en el territorio nacional que incrementan su vulnerabilidad, tales como el hecho de ser mujeres, quienes en muchos casos son de origen indígena, su condición de migrantes, su edad temprana y en ocasiones el ser domésticas de tiempo completo, lo que las hace residir en un hogar ajeno limitando su independencia. Cabe señalar que las condiciones de desigualdad y abusos hacia las trabajadoras domésticas mexicanas no se limitan a aquellas que habitan en el país. En Estados Unidos de Norteamérica Burnham y Theodore (2012, p. 41) señalan que alrededor del $95 \%$ de las personas que se desempeñan como trabajadores domésticos en ese país son mujeres, el 78\% es de origen extranjero y el $56 \%$ no cuenta con ciudadanía, lo que las ubica fuera de la ley y por lo tanto, no acreedoras a protección legal laboral. Como se sabe, México es "exportador" de grandes 
cantidades de mano de obra hacia el país vecino, principalmente caracterizada como migración ilegal.

Por otro lado, puesto que las labores domésticas han sido históricamente desarrolladas por mujeres de forma no remunerada, esta actividad no se encuentra contemplada dentro de la definición convencional del trabajo por lo que, "sigue siendo una actividad relegada al ámbito de la privacidad y a merced de la explotación y de los abusos" (Parella, 2003, p. 122). Al respecto, Magliano (2017, p. 8) afirma que la falta de reconocimiento de los derechos laborales de las trabajadoras del hogar por parte de sus empleadores, se origina en el antecedente de que este tipo de trabajos surgieron como parte de relaciones interpersonales y no de una relación laboral regulada, basándose más en la "voluntad" y "confianza" mutua, que en un contrato establecido.

\section{Lo que señalan los resultados de ENADIS (2010)}

De acuerdo con el Consejo Nacional para Prevenir la Discriminación (CONAPRED, 2011, pp. 32-44), los resultados generados a través de la Encuesta Nacional sobre Discriminación en México (ENADIS, 2010), arrojaron que el 38.8\% de las mujeres encuestadas consideran que el principal problema para este grupo laboral es la percepción salarial baja, mientras que el $19.3 \%$ señalaron como segundo lugar aspectos relacionados con el trato (abusos, maltratos, humillaciones y discriminación). 
Respecto a las condiciones de trabajo, los resultados ponen en evidencia que nueve de cada diez mujeres trabajadoras del hogar no cuentan con un contrato laboral formal, lo cual de inicio ya brinda la pauta para la omisión de derechos laborales para ellas. Estas omisiones se presentan en diferentes formas. En cuanto al horario laboral, en la mayoría de los casos las trabajadoras dedicadas al servicio doméstico laboran de 5 a 6 días, aunque en menor escala $(6.5 \%)$, llegan a trabajar hasta siete días a la semana, lo que implica que no gozan de por lo menos un día de descanso semanal, mientras que el $47.4 \%$ refirió trabajar durante todo el día. Dentro de la falta de derechos laborales que más se presentaron en la muestra estudiada, estuvieron la falta de prestaciones como servicios médicos, vacaciones, aguinaldos y horarios fijos.

A pesar de todo esto, el $54.1 \%$ de la muestra no considera que en México no se respetan los derechos de las trabajadoras del hogar. Es decir, parecieran no estar conscientes de sus derechos y de la omisión que sus patrones hacen de los mismos. El CONAPRED (2011, p. 50) señala que existen características en el ámbito laboral que favorecen estos resultados, tales como el aislamiento e invisibilidad que se presenta y que deriva en la poca o nula garantía en sus derechos como trabajadoras. Así mismo, el grado de vulnerabilidad se incrementa cuando el trabajo del hogar está feminizado. Esto coincide con los hallazgos realizados por Barrón (2013, p. 111) al sur de México, donde se establece que por cada trabajador masculino que posee un empleo identificado como vulnerable, -es decir, trabajadores por cuenta propia o asalariados con condiciones 
laborales precarias-, 1.36 mujeres en Colima y 1.10 mujeres en $\mathrm{Vi}$ -

lla de Álvarez, presentan la misma condición.

En cuanto al trato con respeto, el $79.9 \%$ considera que ha sido tratado con respeto en su trabajo, sin embargo, si se toma en cuenta la respuesta "a veces", se puede decir que dos de cada diez trabajadoras del hogar han vivido la experiencia de no ser tratadas con respeto en el ámbito laboral. De igual forma, de enero a octubre del 2010, el 12.7\% de las trabajadoras del hogar que dejaron su empleo, lo hicieron por percibir malos tratos hacia ellas en su lugar de trabajo.

\section{Metodología}

Considerando que tanto la desigualdad laboral como el maltrato por parte de patrones hacia las empleadas del hogar son situaciones que violentan y merman la calidad de vida de dichas trabajadoras, se realiza el presente estudio como un avance en los trabajos de investigación de la red "Evaluación e intervención en factores de riesgos psicosociales", cuyo objetivo principal es prevenir las conductas que inciden sobre la salud física, social y emocional de la población trabajadora, en este caso, de las mujeres trabajadoras del hogar.

Para el desarrollo del presente, la fuente de la cual se obtuvieron los datos a analizar fue generada por el Consejo Nacional para Prevenir la Discriminación (CONAPRED, 2010), por ser la base de datos de acceso abierto más reciente a nivel nacional. La informa- 
ción tomada se derivó del factor 8 de la encuesta respecto al trabajo doméstico.

Como primer paso se determinaron las variables de estudio. Estas se clasificaron en dos dimensiones que caracterizan la relación de trabajo y que pueden incidir en la percepción de las integrantes de la muestra respecto al trato recibido por sus empleadores. La primera se denominó características contractuales del trabajo y la segunda características del trato. Ambas se clasificaron como variables independientes.

Figura 1. Dimensiones y variables independientes de los modelos

\begin{tabular}{|c|c|c|c|}
\hline & Factor & Variable & Pregunta \\
\hline \multirow[t]{5}{*}{1} & \multirow{5}{*}{$\begin{array}{l}\text { Características } \\
\text { contractuales } \\
\text { del trabajo }\end{array}$} & Horario & ¿Tiene horario fijo? \\
\hline & & Aguinaldo & ¿Le dan algún aguinaldo? \\
\hline & & Vacaciones & ¿Le dan vacaciones? \\
\hline & & Seguro & ¿Cuenta con seguro médico? \\
\hline & & Pago & ¿Le pagan con regularidad? \\
\hline \multirow[t]{4}{*}{ II } & \multirow[t]{4}{*}{$\begin{array}{l}\text { Características } \\
\text { del trato }\end{array}$} & Comer & $\begin{array}{l}\text { ¿Come usted lo mismo que la } \\
\text { familia? }\end{array}$ \\
\hline & & Teléfono & ¿Puede usar el teléfono? \\
\hline & & Llave & $\begin{array}{l}\text { ¿Tiene llave de la puerta de } \\
\text { entrada? }\end{array}$ \\
\hline & & Permiso & $\begin{array}{l}\text { ¿Le dan permiso cuando necesita } \\
\text { salir? }\end{array}$ \\
\hline
\end{tabular}

Fuente: elaboración propia con información del ENADIS generada por el CONAPRED (2010). 
Las preguntas que miden las variables independientes que corresponden a las características contractuales del trabajo y a las características del trato, se detallan en la Figura 1. Todas las variables son dicotómicas con respuestas sí o no y el nivel de medición de cada una de ellas es nominal.

Para la variable dependiente se consideró la pregunta sobre trato con respeto, la cual fue dicotómica, con nivel de medición nominal con respeto y sin respeto. Una vez definida las variables, se aplicó una regresión logística binaria multivariada, para obtener los modelos de estudio y estimar la probabilidad de tener un trato con respeto en función a cada una de las variables propuestas.

\section{Resultados}

Los resultados respecto a las características de la muestra fueron los siguientes. La muestra estuvo conformada por un total de 404 mujeres trabajadoras domésticas de la República Mexicana, con una edad media de 41.5 años y una desviación estándar de 12.4 años. La cantidad de féminas que sabe leer o escribir fueron 373 (92.3\%), mientras que 7 (1.9\%) asistían a la escuela. El estado civil de las mujeres se distribuye como: 134 (33.2\%) casadas, 79 (19.6\%) solteras, 71 (17.6\%) separadas, 54 (13.4\%) viven con su pareja, 41 (10.1\%) viudas, $23(5.7 \%)$ divorciadas y $2(0.5 \%)$ no contestó. 
En lo concerniente a la percepción salarial, la distribución se muestra en la Figura 2.

Figura 2. Distribución de las trabajadoras según el salario semanal

\begin{tabular}{|l|l|}
\hline Salario (pesos Mx) & Cantidad de trabajadoras \\
\hline A lo más \$400 & $110(27.2 \%)$ \\
\hline Entre \$401 y \$600 & $113(28.0 \%)$ \\
\hline Entre \$601 y \$800 & $73(18.1 \%)$ \\
\hline Más de \$800 & $68(16.8 \%)$ \\
\hline Casos perdidos & $40(9.9 \%)$ \\
\hline
\end{tabular}

Fuente: elaboración propia con información de ENADIS generada por el CONAPRED (2010). 
En cuanto a las prestaciones laborales, los resultados se presentan en la Figura 3.

Figura 3. Distribución de las trabajadoras según el tipo de trabajo

\begin{tabular}{|l|l|l|}
\hline Variable & Pregunta & Total \\
\hline Horario & ¿Tiene horario fijo? & Sí $184(45.5 \%)$ \\
& & No $180(44.6 \%)$ \\
& & A veces $38(9.4 \%)$ \\
NS $\quad 2(0.5 \%)$
\end{tabular}

Fuente: elaboración propia con información de ENADIS generada por el CONAPRED (2010). 
Los resultados manifiestan que existe desigualdad laboral en este grupo de trabajadoras, generada a partir de jornadas laborales arbitrarias, falta de prestaciones sociales y salarios poco competitivos, ya que se puede constatar que más del $27 \%$ de la población estudiada tiene un ingreso no mayor a los $\$ 400.00$ pesos por semana. Esto es inferior al salario mínimo establecido por el Consejo Nacional de Salarios Mínimos (CONASAMI, 2010, p. 1), para ese año, equivalente a $\$ 54.47$ pesos diarios establecido para actividades generales, tomando como referencia el tabulador más bajo (zona geográfica C).

Así mismo, se encontró que el $44.6 \%$ no cuenta con un horario fijo, el $49 \%$ no recibe aguinaldo, el $51.7 \%$ no tiene derecho a vacaciones pagadas, el $86.9 \%$ no tiene cobertura médica como trabajadora y el 3\% no recibe su sueldo con regularidad. Con respecto al trato brindado por sus patrones, de acuerdo con la percepción de las empleadas del hogar, se encontró que 303 trabajadoras, correspondientes al $75 \%$ de la muestra consideran que son tratadas con respeto, mientras que 96 (23.7\%) manifiestan que no son tratadas con respeto, al menos en ocasiones. Por último, 5 encuestadas (1.2\%) no supo definir si ha sido tratada o no con respeto, o no respondió.

Respecto a la generación de los modelos estadísticos I y II, para determinar la asociación entre variables, se obtuvieron los resultados que se muestran en la Figura 4 a partir de una regresión logística binaria multivariada, donde la variable dicotómica dependiente del nivel de medición nominal fue el tipo de trato; con respeto y sin respeto. 
YOLANDA VELÁZQUEZ NARVÁEZ / FABIOLA PEÑA CÁRdENAS / LUCÍA RUÍZ RAMOS

Figura 4. Resultados de los modelos de probabilidad

\begin{tabular}{|c|c|c|c|c|c|c|c|}
\hline Modelo & Variables & $\begin{array}{l}\text { Coeficiente } \\
\text { (B) }\end{array}$ & $\begin{array}{l}\text { Error } \\
\text { estándar }\end{array}$ & Wald & g.l. & p-valor & $\operatorname{Exp}(B)$ \\
\hline \multirow[t]{6}{*}{1} & Horario & 0.068 & 0.270 & 0.064 & 1 & 0.801 & 1.10 \\
\hline & Aguinaldo & 0.732 & 0.325 & 5.052 & 1 & 0.025 & 2.10 \\
\hline & Vacaciones & -0.534 & 0.367 & 2.115 & 1 & 0.587 & 0.60 \\
\hline & Seguro & -0.395 & 0.666 & 0.351 & 1 & 0.674 & 0.70 \\
\hline & Pago & -0.901 & 0.490 & 3.378 & 1 & 0.066 & 0.41 \\
\hline & Constante & 1.638 & 0.692 & 5.611 & 1 & 0.018 & 5.15 \\
\hline \multirow[t]{5}{*}{ II } & Comer & -0.510 & 0.328 & 2.416 & 1 & 0.120 & 0.60 \\
\hline & Teléfono & 0.919 & 0.258 & 12.649 & 1 & 0.000 & 2.50 \\
\hline & Llave & -0.341 & 0.275 & 1.542 & 1 & 0.214 & 0.71 \\
\hline & Permiso & 0.725 & 0.315 & 5.295 & 1 & 0.021 & 2.06 \\
\hline & Constante & 0.479 & 0.376 & 1.622 & 1 & 0.203 & 1.62 \\
\hline
\end{tabular}

Fuente: elaboración propia. (g.l. = grado de libertad, $\operatorname{Exp}(B)=$ Exponencial de $B$ ). 
Como se aprecia en la Figura 4, para el modelo I correspondiente a la dimensión características contractuales de trabajo, se encontró que la única variable asociada al trato con respeto fue aguinaldo. En otras palabras, una trabajadora que reciba aguinaldo tiene 2.1 veces más probabilidades de percibir un trato con respeto que una colega que no lo reciba. Esto puede deberse a que, dada la nulidad de protección laboral con que cuentan las trabajadoras de este sector productivo, el recibir una gratificación económica adicional a su sueldo, suele ser considerado como causa de la buena voluntad de su empleador, y esto tiene que ver más en este caso, con una actitud de gratitud y reconocimiento hacia el trabajo realizado durante el año, que con una obligación legal.

Respecto al modelo II, correspondiente a las características de trato, las variables asociadas al trato con respeto son dos, 1) la libertad de utilizar el teléfono de casa cuando lo requiere y disponer de permiso para salir si lo necesita. Es decir, una trabajadora que pueda usar el teléfono de la casa donde trabaja cuando lo requiere, tiene 2.5 veces más probabilidades de sentirse tratada con respeto que una empleada a la que no se le permita usarlo. De la misma forma, una empleada que obtenga permiso de salir cuando lo necesite tiene el doble (2.06) de probabilidades de tener una percepción de ser tratada con respeto que una colega a quien no se le otorgan permisos.

Aunque no se puede afirmar con certeza las razones de estas asociaciones, puede asumirse que ambas variables se refieren al grado de libertad que recibe la empleada para atender asuntos pri- 
vados en su contexto familiar, de pareja o personal (de salud, educativos, legales, etc.), dentro del horario laboral establecido, lo que implica cierto grado de empatía de parte de sus empleadores.

\section{Consideraciones finales}

En México existen esfuerzos por reconocer la igualdad de género en el ámbito laboral, a pesar de ello, la desigualdad y discriminación hacia la mujer trabajadora sigue siendo un problema latente. En este sentido, un grupo altamente vulnerable es el de las trabajadoras del hogar, las cuales son víctimas de la negación de derechos como trabajadoras incidiendo en salarios y prestaciones muy por debajo de otras actividades productivas, al prevalecer una casi nula protección jurídica hacia ellas (Ghai, 2003, p. 125; Anguiano y Ortíz, 2013, p. 97). Por lo tanto, estas características de trabajo son opuestas a lo denominado trabajo decente (Barrón, 2013, p. 109). Esta problemática se incrementa cuando la actividad laboral es feminizada (CONAPRED, 2011, p. 50), y se agudiza cuando dichas mujeres pertenecen a grupos en desventaja social (Daeren, 2000, p. 8; Durin, 2013, p. 104), de tal forma que estas condiciones de trabajo generan que las mujeres sean blanco fácil para otro tipo de victimización (Alonso et al., 2006, p. 156), como la violencia explícita, manifestada por agresiones, acoso y humillaciones.

No obstante, llama la atención que a pesar de ello, el 75\% de las trabajadoras que integran la muestra consideran que son trata- 
das con respeto, mientras que el $54.1 \%$ de la muestra incluida en la ENADIS (2010) no considera que en México no se respeten los derechos de las trabajadoras del hogar. Esto hace cuestionar qué tan conscientes están las trabajadoras del hogar sobre sus derechos y qué características de la relación trabajadora-patrón asocian a dicho trato. Por lo tanto, se considera que el mayor aporte del presente trabajo consistió en estimar la probabilidad de que la empleada doméstica perciba un buen trato por parte de sus patrones, considerando aspectos tanto del grado de libertad y confianza percibidos por la empleada, y características contractuales de trabajo, llegando a la conclusión de que la percepción que tienen las trabajadoras del hogar con relación al trato con respeto que reciben por parte de sus empleadores no se asocia directamente a las características contractuales de trabajo, sino, al grado de libertad percibido para atender situaciones personales durante su jornada laboral.

Se considera que es necesario que en México se impulsen y fortalezcan programas enfocados a las mujeres trabajadoras, sobre todo dentro del ramo informal, que se brinde información sobre equidad de género y se ayude a identificar situaciones que las hacen víctimas de actos de discriminación, de tal forma que estas acciones coadyuven a su empoderamiento dentro de las diferentes esferas sociales donde se desempeñan, a la par que se fortalezca el marco jurídico respecto al reconocimiento de sus derechos laborales. 
YOLANDA VELÁZQUEZ NARVÁEZ / FABIOLA PEÑA CÁRdENAS / LUCÍA RUÍZ RAMOS

\section{Bibliografía}

Alonso, M. M., Musayon, F. Y., SCherlowski, H. M. y GÓMEZ, M. V. (2006).

Consumo de drogas y violencia laboral en mujeres que trabajan, un estudio multicéntrico: México, Perú, Brasil. Revista Latino-Americana de Enfermagem, 14(2), 155-162.

Anguiano, A. y Ortíz, R. (2013). Reforma laboral en México: precarización generalizada del trabajo. El Cotidiano, (182), 95-104.

Barrios, A. Y. y Barrios, O. A. (2016). Participación femenina en el mercado laboral de México al primer trimestre de 2016. Economía Actual, 9(3), 41-45.

BARRÓN, M. A. (2013). Trabajo decente, trabajo vulnerable y trabajo precario entre la población ocupada de los municipios de Colima y Villa de Álvarez del Estado de Colima, México. Una visión de género. Revista de investigación y divulgación sobre los estudios de género, (14), 99-118.

Burnham, L. y TheOdore, N. (2012). Home Economics. The Invisible and Unregulated World of Domestic Work. New York: National Domestic Workers Alliance, Center for Urban Economic Development, University of Illinois at Chicago and Data Center.

CONASAMI. (2010). Salarios Mínimos. México: CONASAMI. Recuperado de http://www.conasami.gob.mx/pdf/tabla_salarios_minimos/ 2010/01_01_2010.pdf

CONAPRED (2010). Encuesta Nacional sobre Discriminación en México 2010 - ENADIS. Base de datos en SPSS. México: CONAPRED. Recuperado de https://datos.gob.mx/busca/dataset/encuesta-nacionalsobre-discriminacion-en-mexico-2010-enadis-2010-base-de-datos-en-spss 
(2011). Encuesta Nacional sobre Discriminación en México. Resultados Generales. México: CONAPRED. Recuperado de http:// www.conapred.org.mx/userfiles/files/Enadis-2010-RG-Accss-002.pdf

DAEREN, L. (2000). Género en la migración laboral internacional en América Latina y El Caribe. Pautas para "buenas prácticas" en la formulación de políticas y programas dirigidos a trabajadoras y trabajadores migrantes. OIT. Santiago, Chile. Recuperado de https://www.cepal.org/mujer/ proyectos/gtz/publicaciones/word.../migracionlaboral.doc

DuRIN, S. (2013). Servicio doméstico de planta y discriminación en el área metropolitana de Monterrey. Relaciones, (134), 93-129.

GHAI, D. (2003). Trabajo decente. Concepto e indicadores. Revista Internacional del Trabajo, 122 (2), 125-160.

Hidalgo, A. (2016). El acceso a la justicia laboral de las personas trabajadoras migrantes nicaragüenses en Costa Rica. Revista Internacional y Comparada de Relaciones Laborales y Derechos del Empleo, 4(3) 1-18.

Horbath, J. E. (2008). La discriminación laboral de los indígenas en los mercados urbanos de trabajo en México: revisión y balance de un fenómeno persistente. En: F. García (Ed.), Identidades, etnicidad y racismo en América Latina (pp. 121-158). Quito: Colección 50 años FLACSO.

y Garcia, A. (2014). Discriminación laboral y vulnerabilidad de las mujeres frente a la crisis mundial en México. Economía, Sociedad y Territorio, 14(45), 465-495.

INEGI. (2017). Estadísticas a propósito del día internacional de la mujer. Datos nacionales. México: INEGI. Recuperado de http://www.inegi.org.mx/ saladeprensa/aproposito/2017/mujer2017_Nal.pdf. 
YOLANDA VELÁZQUEZ NARVÁEZ / FABIOLA PEÑA CÁRDENAS / LUCÍA RUÍZ RAMOS

LeXartza, L., Chaves, M. J. y CARCEdo, A. (2016). Políticas de formalización del trabajo doméstico remunerado en América Latina y El Caribe. Perú: Organización Internacional del Trabajo.

MAgLiAno, M. J. (2017). Las trabajadoras invisibles: experiencias laborales de mujeres migrantes en Argentina. Revista LAT, (1), 1-23.

MAQUEDA, M. L. (2006). La violencia de género. Entre el concepto jurídico y la realidad social. Revista Electrónica de Ciencia Penal y Criminología, 8(2), 1-13.

MendozA, C. A. y ArmendÁRIz, V. (2017). Seguridad Social para el trabajo del hogar. Serie Derechos laborales de las trabajadoras del hogar en México. $1-13$.

Organización Internacional del Trabajo. (2010a). Camino del trabajo decente para el personal del servicio doméstico: panorama de la labor de la OIT. Francia: Organización Internacional del Trabajo. Recuperado de http://www.ilo.org/wcmsp5/groups/public/—dgreports/—gender/ documents/publication/wcms_142907.pdf

(2010b). Trabajo decente para los trabajadores domésticos. Ginebra: Organización Internacional del Trabajo. Recuperado de http:// www.ilo.org/wcmsp5/groups/public/@ed_norm/@relconf/ documents/meetingdocument/wcms_124841.pdf

(2018). Economía informal. Nueva York: Organización Internacional del Trabajo. Recuperado de http://www.ilo.org/ilostat-files/ Documents/description_IFL_EN.pdf

PARELla, S. (2003). Mujer, inmigrante y trabajadora: la triple discriminación. Barcelona: Anthropos Editorial. 
ReYnoso, C. (2011). Nuevas normas internacionales sobre trabajo doméstico. Alegatos, 78, 593-594.

Rodgers, J. (2009). Cambios en el servicio doméstico en América Latina. En M. E. Valenzuela y C. Mora (Eds.), Trabajo doméstico: un largo camino hacia el trabajo decente (pp. 1-305). Chile: Organización Internacional del Trabajo.

Rojas, G. y CONTRERAs, N. (2018). Resistencia activa de las trabajadoras del hogar en México: talleres, aprendizaje y empoderamiento. Revista LAT, Dossier Trabajo, conflictividad y resistencias, (3), 1-37.

SANTILlÁN, I. R. (2016). Precariedad en derechos y reconocimiento del trabajo que se realiza en casa: el caso de México. Revista de Estudios de las Mujeres, 4, 49-71. 